\title{
Brote de cryptosporidiosis en un centro de recuperación nutricional
}

\author{
Dr. Juan Carkos Weitz V.1 , Dr. Renzo Tassara O.1, T.M. Rubén Mercado P.1, \\ Dr. Francisco Santa María P. ${ }^{2}$, Dra. Margarita Maida S. ${ }^{3}$, Dr. Pedro Lorca O. 1 , \\ Prof. Dr. Antonio Atías M. 1
}

\section{Cryptosporidiosis in children of a nutritional care center}

\begin{abstract}
An outbreak of cryptosporidjosis is describod in a nutritional center of Santiago: the finding of the infection in two undernowished in patient children lead to the study and detection of Cyptosporidum sp. in stool specimens of $24.3 \%$ of the children in the same center. Fxarnination of controls from a different center showed a $5.5 \%$ rate of infection $(p<0.01)$. Eight patients suffered acute diarthea, 6 had respiratory symptoms and 6 were asymptomatic. (Key words: Cryptosporidium outbreak. nut ritional tare center).
\end{abstract}

Cryptosporidum sp. es un protozno coccidio que parasita el epitelio gastro-intestinal de mamíferos, causando diarrea aguda, especialmente en animales jóvenes ${ }^{1}$. Además, en algunas aves, afecta el aparato respiratorio. ${ }^{1,2}$

En el ser humano es capaz de producir diartea crónica severa en pacientes inmunocomprome$\operatorname{tidos}^{3}$ y diarrea aguda autolimitada en inmunocompetentes. ${ }^{3,4}$ Las infecciones intestinales por Cryptosporidum sp. se han comunicado en todo el mundo y, en especial, en niños con diarsea aguda. En Europa alcanza una frecuencia del $1,4 \%$ en lnglaterra ${ }^{5}$ a $2 \%$ en Dinamarca ${ }^{6}$; en Australia, 4,1\% ; en Africa (Ruanda) $10,4 \%^{8}$ y en América (Estados Unidos $2.8 \%^{4}$; Costa Rica $4,3 \% 9$; Perú $8,1^{10}$; Venezuela $10,8 \% 11$; y en Chile $6,1 \%{ }^{12}$ ).

Los estudios de los contactos de pacientes con cryptosporidiosis se han efectuado en grupos humanos que trabajan con animales (granjas agrícolas).$^{13}$ en familias que han visitado países de alta endemia ${ }^{14}$ y recientemente, en jardines infantiles ${ }^{15,16,17}$.

El objetivo de este trabajo es comunicar un brote de esta parasitosis en un centro de recuperación nutricional de Santiago, detectađo a partir de dos niños infectados con este protozoo que padecían diarrea aguda.

1. Unidad de Parasitología, Div. Cs. Méd. Occidente, Fac. Medicina. U, de Clike.

2. Dírector Médico Conin Pudahuel.

3. Director Médico Conin Vitacura.

\section{MATERIAL Y METODO}

Fin un estudio anterior ${ }^{12}$, medante la tinción de Ziehl-Neelsen, pesquisamos 11 wasos de cryptosporidiosis, de un total de 180 pacientes encuestados. Todos eran niños con diarrea ayuda severa, acompañada de deshidratación y acidosis 18 . De ellos, 2 lactantes, de 12 y 13 meses te cdad respectivamentc, provenían del centro de recuperación nutricional Conin Pudahuel, a partir de los cuales investigamos cl parásito en dicho contro, (Grupo 1), encuestando a 103 personas: 33 adul tos (32\%) y 70 niños $(68 \%)$, incluidos los dos casos índices. Como grupo control, se escogió a otro centro similar, Conin Vitacura (Grupo ID), compuesto de 76 individuos: 22 adultos. (28,9\%) y 54 niños $(71,1 \%$. La distribución por edad y sexo de los niños de ambos centros nutricionales son comparables: edad promedio 10,9 mescs (Grupo I) y $10,1 \mathrm{~m}$ (Grupo li), sexo masculino $54,3 \%$ y $53,9 \%$ respectivamente.

A los 55 adultos se les consulto pot síntomas digestiwos, y se revisaron las fichas clínicas de los 124 niños, completando datos de edad, sexo, estado nutricional $y$ sintonas. Conjuntamente con ello, se Jes solicitó una muestra seriada de deposiciones en fràscos con formol-sal, que se procesaron, sin conocer su procedencia, según al método de Telemann modificado (MTM) ${ }^{19}$ y además se tomó, del sedimento, una gota con la cual se realizó un frotis, que fue teñida con técnica de Ziehl-Neelsen ( $Z N$ ). De las muestras positivas al $Z N$ sc efectuaron dos frotis más, sometiéndolos a tinciones con Aureamina y Aureamina-Rodamina20, para tener una confirmación por medio de tres métodos de diagnóstico.

\section{RESULTADOS}

Los adultos de ambos grupos fueron asintomáticos y Ja tinción de Ziehl-Neelsen fue negativa para Cryptosporidium en todos (Tabla 1). El MTM fue positivo en $17(51,5 \%)$ del Grupo I; de ellos, 7 tenían parásitos en las heces. En 8 sujetos 
del Grupo II (36,6\%) los resultados del MTM fueron positivos, en la mayoria con comensales.

La Tabla 2 muestra el resultado de] examen con Ziehl-Neelsen en las deposiciones de los niños de ambos centros. En el grupo de estudio, $17(24,3 \%)$ tenían Cyptosporidium sp. en sus deposiciones. En el grupo control, $3(5.5 \%)$ dieron resultados $Z N$ positivos, diferencia que es estadísticamente significativa $(\mathrm{p}<0,01)$. En estos 20 casos también fueron positivos los resul. tados con las tinciones Aureamina y Aureamina - Rodamina.

En la Tabla 3 'se observa la distribución de los nif̂os según edad. De los 17 casos positivos del Grupo 1, cuatro eran menores de 6 meses, 5 entre 6 y 12 meses, y 8 entre 12 y 18 meses. Los 3 casos del Grupo II tenían entre 6 y 18 meses. Ningún niño infectado es mayor de 18 meses.

Tabla 1.

Detección de Crvprosporidium sp. y otros enteroparásitos en adultos de dos centros nutricionales

\begin{tabular}{lccccc}
\hline & \multicolumn{2}{c}{ GRUPOI } & \multicolumn{2}{c}{ GRUPO II } \\
\cline { 2 - 3 } \cline { 5 - 6 } Técnica. & n/total & $\%$ & & & \\
\hline $\begin{array}{l}\text { Z Neelsen } \\
\text { Negativo }\end{array}$ & $33 / 33$ & 100 & & $22 / 22$ & 100 \\
$\begin{array}{l}\text { Telemann Mod. } \\
\text { Positivo }\end{array}$ & $17 / 33$ & 51,5 & $8 / 22$ & 36,3 \\
\hline
\end{tabular}

Grupo I: Personal del centro nutricional Pudahuel (pacientes)

Grupo II: Personal del centro nutriciona] Vitacura (controles)

Tabla 2.

Detección de Cryptosporidium sp. por técnica dẽ Zichl-Neclsen en nijios de dos centros nutricionales

\begin{tabular}{|c|c|c|c|c|c|}
\hline \multirow[b]{2}{*}{ Resultado } & \multicolumn{2}{|c|}{ GRUPO I } & \multicolumn{2}{|c|}{ GRUPO II } & \multirow[b]{2}{*}{$\mathrm{p}$} \\
\hline & 17 & $\%$ & $\pi$ & $\%$ & \\
\hline Positivo & 17 & 24,3 & 3 & 5,5 & \\
\hline Negativo & 53 & 75,7 & 51 & 94,5 & \\
\hline Total & 70 & 100 & 54 & 100 & \\
\hline
\end{tabular}

Grupos I y Il como en Tabla 1.
Tabla 3.

Cryptosporidiosis en niños de dos centros nutricionales, distribución según edad.

\begin{tabular}{lcc}
\hline Edad & $\begin{array}{c}\text { Grupo I } \\
\text { Positiwos } / \text { Total }\end{array}$ & $\begin{array}{c}\text { Grupo lI } \\
\text { Positivos Total }\end{array}$ \\
\hline$<6$ meses & $4 / 10$ & $0 / 10$ \\
$6-12 \mathrm{~m}$. & $5 / 26$ & $2 / 32$ \\
$12-18 \mathrm{~m}$. & $8 / 28$ & $1 / 7$ \\
$18 \mathrm{~m}$. y mas & $0 / 6$ & $0 / 5$ \\
\hline Total & $17 / 70$ & $3 / 54$ \\
\hline
\end{tabular}

Grupus I y Il como en Tabla 1.

Según el estado de nutrición la muestra de Conin Pudahuel estaba formada por 5 eutróficos, 29 desnutridos grado 1,27 grado II y 9 grado III. En Conin Vitacura, 9 eran eutróficos, 14 desnutridos grado I, 23 grado II y 8 grado [II. Todos los casos de Conin Pudahuel fueron detectados en niños desnutridos: 8 grado I, 6 grado II y 3 grado III. En el grupo control, 2 niños eran eutróficos y uno desnutrido grado II.

Los sintomas digestivos de los niños encuestados se analizan en la Tabla 4. En el Grupo I, 15 $(21,4 \%)$, padecian de diarrea aguda en el momento del examen; de ellos. $8(53,3 \%)$ tenian Cryptosporidium sp. en sus heces. En el Grupo Il, $7(12,9 \%)$ sufrian de diarrea aguda, todos $Z N$ negativos. Los 12 casos restantes con esta protozoosis ( 9 del Grupo I y 3 controles), no tenian síntomas gastrointestinales, pero 6 cursaron con molestias respiratorias (broncuitis aguda, bronquitis obstructivas) y 6 fueron totalmente asintomáticos.

Los centros encuestados cuentan con 8 y 6 salas respectivamente para la ubicación de los niños. Los casos positivos fueron detectados en la totalidad de las salas del Grupo I y sólo en 2 salas del Grupo II.

El MTM fue positivo en 10 niños $(14,3 \%)$ de Grupo 1 ( todos con parásitos) y en $8(14,8 \%)$ del grupo control ( 6 con parásitos y 2 con comensa. les). Del total de niños con cryptosporidiosis, sólo uno estaba infectado con otro parásito (Giardia lamblia).

Tabla 4.

Frecuencia de Cryprosporiditum sp. en riños con diarrea y asintomáticos

\begin{tabular}{lcccc}
\hline Sintomas & \multicolumn{2}{c}{$\begin{array}{c}\text { Grupo I } \\
\text { No }(\mathrm{ZN+}) / \text { Total } \%\end{array}$} & \multicolumn{2}{c}{$\begin{array}{c}\text { Grupo Il } \\
(\mathrm{ZN}+) / \text { Total } \%\end{array}$} \\
\hline Diarrea & $8 / 15$ & 53,3 & $0 / 7$ & 0 \\
Asintomátio & $9 / 55$ & 16,4 & $3 / 47$ & 6,3 \\
\hline Total & $17 / 70$ & $\mathbf{2 4 , 3}$ & $3 / 54$ & 5,5 \\
\hline
\end{tabular}

$\mathrm{ZN}=$ tinción de Ziehl-Neelsen

Grupos I y II como en Tabia 1. 


\section{DISCUSION}

Nuestra investigación se realizó a partir de dos lactantes desnutridos, que fueron hospitalizados en el Servicio de Pediatría del Hospital San Juan de Dios de Santiago por diarrea aguda severa. A] estudiar el centro nutricional de donde procedian, $24,3 \%$ de los niños atendidos en el tenian Oryptosporidium $s p$. en sus deposiciones, cifra estadísticamente significativa $(p<0,01)$ si se la compara con el grupo control. Estos hallazgos demuestran la existencia de un brote de cryptosporidiosis en ese centro. El análisis de las muestras de deposiciones de los adultos que alli trabajan, no mostró infección por este parásito. Recientemente, se ha comunicado la posibilidad de efectuar estudios serológicos, con pesquisa de anticuerpos anti-Cryptosporidium, en los contactos de pacientes con criptosporidiosis ${ }^{21}$. Probablemente en el futuro, este examen podría ayudar a la detección de un número mayor de casos e incluso, orientar acerca del punto de partida de la infección en personas afectadas.

Todos los niños parasitados con Cryptosporidium $s p$. eran menores de 18 meses de edad; los 17 casos de Conin Pudahuel y uno del grupo control eran desnutridos y dos casos del grupo control fueron detectados en niños eutróficos.

Sólo 8 de los niños presentaban diarrea aguda; los 12 restantes no habían tenido ni tuvieron molestias gastroinfestinales en relación con la fecha de la encuesta, seis de ellos, presentaron síntomas respiratorios (bronquitis aguda $y$ bronquitis obstructiva) lo que ha sido descrito en otros estudios 22 . Probablemente el compromiso respiratorio se deba a la presencia de parásito en ese sistema. pues el Cryptosporidium $s p$. ha sido aislado de la vía respiratoria por algunos autores $^{23}$ y por nosotros ${ }^{24}$. Los otros 6 niños fueron totalmente asintomáticos, forma de presentación descrita ocasionalmente en la literatura $3,13,17$.

La distribución de los casos en todas las salas del centro nutricional afectado muestra la gran facilidad de transmisión de este protozoo, en especial por medio del contacto persona-persona, lo que puede significar en el futuro un verdadero problema epidemiológico en jardines infantiles, escuelas, hospitales y otras institu. ciones similares.

Estos resultados, que muestran el primer brote descrito de cryptosporidiosis en nuestro país, ocurrido en niños desnutridos, con un número importante de casos asintomáticos, señalan la necesidad de continuar los estudios sobre la cryptosporidiosis, con el fin de conocer mejor sus formas de presentación clínicas, así como sus aspectos epidemiológicos lo que permitiria im. partir normas de manejo y control adecuados.

\section{RESUMEN}

Se describe un brote de cryptosporidiosis en un centro nutricional de Santiago. A partit de 2 lactantes desnutridos con esta parasitosis, se detectó que en Conin Pudahuel, el 24,3\% de los niños tienen Cryptosporidium sp. en sus deposiciones, en cambio en el grupo control el 5.5\% está infectado con este parásito $(\mathrm{p}<0,01)$.

De estos pacientes, 8 padecían de diartea aguda, 6 sintomas respiratorios y 6 eran asintomáticos.

\section{REFERENCIAS}

1. Tzipori, S.: Cryptosporidiosis in animals and lıumans. Microbjol. Rey. 47: 84, 1983.

2. Itakura, C., Nakamura, $H_{\text {. }}$ timemura, $T$. Goro, M.: Ultrastructure of cryptosporidial life cycle in chicken host cells. Avtn. Pathol. 14: 237, 1985.

3. Navin. T., and Juranek. D.: Cryptosporidiosis: clinical, epidemiologic and parasitologic review. Rev. Infect. Dis. 6: $313,1984$.

4. Wolfoun, $I$, Richter, $J$, waldron. M., Weber, $D$. Mc Carthy, D. and Hopkins, C.: Cryptusporidjosis in immunocompetent patients. N. Engl. J. Med. 312: $1278,1985$.

5. Casemore, D. and Jackson, B.: Sporadic cryptosporidiosis in children. Lancet. 2: 679, 1983.

6. Hotten-Andersen, W, Gerstoft, $J$., Henriksen, $S$ and Pedersen, N.: Prevalence of Cryptosporidium among patients witls acute enteric infection. $J$. Infect. 9: 277, 1984.

7. Tzipori, S., Smith, M. Birch, C., Bornes, G. and Bishop, R.: Cryptosporidiosis in hospilal patients with gastroenteritis. Am. J. Trop. Med. Hyg. 32: 931,1983

8. Bogaerts, J, Lepage, P., Rourroy, D. and Vandepitte, J.: Cryptosporidium spp. a frequent cause of diarrhea in Central $\Lambda$ frica. J. Cin. Microbiol. 20: 874,1984 .

9. Mata, L., Bolaños, H., Pizarro, D. and Vives, $M$. Cryptosporidiosis in children from highland Costa Rica rural and wban areas. Am. J. Trop. Med. Hy. 33: 24, 1984.

10. Novin, T.: Cryptosporidiosis in humans: seview of recent epidemiolagic studies. Fur. I. Epydemiol. 1: 77.1985 .

11. Pétez-Schoel, I., Boher, Y, Mata, L., Pérez, M. and Tapia, $F .:$ Cryptosporidiosis in Venezuelan children with acute dianhea. Am. J. Trop. Med. Hyg. 34: 721,1985

12. Weit 2, J.C., Mercado, R., Tassara, R, Espinoza, M. Lorca, P., Herskovic, P, y Atias, A.: Frecuencia de Crypiosporidium sp. en pacientes hospitalizados, con síntomas digestivos. Rev. Chil. Pediatr. 57:10, 1986.

13. Current, W., Reese, N., Errst, J., Baley, w, Heyman, $M$, Weinstein, $W$ : Human cryptospori. diosis in immunocompetent and immunodeficient persons. Studies of an outbreak and experimcntal transmission. N. Engl. J. Med. 308: 1252, 1983.

14. Soave, R., Ma., P.: Cryptosporidiosis. Traveler's diatrhea in two families. Arch. Intern. Med, 145: $70,1985$. 
15. Alpert, G., Bell, L., Kirkpatrick, C., Buantck, L. Campos, J., Friedman. II., Plotkin, S.: Cryptospotidiosis in a day-care center. N. Fngl, J. Med. 31 I : $860,1984$.

16. Center for Disedse Control (CDC).: Cryptosporidiosis among children attending day-care centersGeorgia, Pennsylva nia. Michigan, Calitor nia, New Mexico. MMWR. 33: 599, 1984.

17. Taylor, J., Perdue, J., Tingley, D., Gustofson. T, Patserson, M., Reed, $L$. Cryptosporidiosis outbreak in a day care cente. Amer, J, Dis. Child 139: $1023,1985$.

18. Tassara, R, weitz. J C., Mercado, R., Espinoza, M., Atios, A,: Crypto roridium sp.: caructeristicas clínicas en 11 casos, Segundo Conyeso de InfectoIogía. Santiago, Octıbre, 1985.

19. Doren, G., Galdat*es, M., Silva, R.: Algunas cons;deraciones sobri al rendimiento de las téunicas de diagnóstico de enteroparasitosis. Bol. Chil. Parasitol. 13: 42, 1958 .

20. Mercado, R., weitz, J.C., Tassaro, R., Atís, A,: T.l diagnóstico de laboratorio de la cryptosporidiosis. Bol. Hosp San Juan de Dios. 33: 87, 1986.

21. Kouh, K. Phillips, D., Aber, R., Current, w.: Cryptosporidiosis in hospital personnel. Ann. Intern. Med. 102: 593, 1985.

22. Hort, C., Baxby, D., Blundel, $N_{.:}$Gastrounteritis duc to Cryptosporidium: i prospective survey in a children's hospital. J. Infect. 9: 264, 1984.

23. Forgacs, P., Tarshis, A., Ma., P., Federman, $M$. Mele. L.. Silverman, $M .$, Shea, $J$. . Intestimal and bronchial cyptosporidiosis in an immunodefïcient homosexual man. Ann. Intern, Med, 99: 793, 1983.

24. Weitz, J.C., Tassara, R., Muñoz, P.. Mercado, R., Atias, $A$. Cryptosporidiosis respirator ja. (Cartil). Rev Med. Chile. (en prensin). 